EGU2020-19310

https://doi.org/10.5194/egusphere-egu2020-19310

EGU General Assembly 2020

(c) Author(s) 2020. This work is distributed under

the Creative Commons Attribution 4.0 License.

\title{
Participation of women scientists in ESA Solar System missions: an historical trend
}

Arianna Piccialli ${ }^{1}$, Julie A. Rathbun ${ }^{2}$, Anny-Chantal Levasseur-Regourd ${ }^{3}$, Anni Määttänen ${ }^{3}$, Anna Milillo ${ }^{4}$, Miriam Rengel ${ }^{5}$, Alessandra Rotundi ${ }^{4}$, Matt Taylor ${ }^{6}$, Olivier Witasse ${ }^{6}$, Francesca Altieri ${ }^{4}$, Pierre Drossart ${ }^{7}$, and Ann Carine Vandaele ${ }^{1}$

${ }^{1}$ Belgian Institute for Space Aeronomy (BIRA-IASB), Planetary Aeronomy, Uccle, Brussels, Belgium

(arianna.piccialli@aeronomie.be)

${ }^{2}$ Planetary Science Institute, Tucson, USA

${ }^{3}$ LATMOS/IPSL, Sorbonne Université, UVSQ, CNRS, Paris, France

${ }^{4}$ INAF, Istituto di Astrofisica e Planetologia Spaziali, Italy

${ }^{5}$ Max-Planck-Institut für Sonnensystemforschung, Göttingen, Germany

${ }^{6}$ ESA, ESTEC, Keplerlaan 1, 2201 AZ Noordwijk, The Netherlands

${ }^{7}$ LESIA, Observatoire de Paris, CNRS, Sorbonne université, Univ. Denis Diderot, F-92195 Meudon, France

We analyzed the participation of women scientists in 10 ESA (European Space Agency) Solar System missions over a period of 38 years [1]. Being part of a spacecraft mission science team can be considered a proxy to measure the "success" in the field. Although the number of female scientists in the field has been constantly increasing in Europe, we did not observe a similar increase in their participation in ESA Solar System missions. Participation of women in PI (Principal Investigators) teams varied between 4 and $25 \%$, with several missions with no women as PI. The percentage of female scientists as Co-I (Co-Investigators) is always less than $16 \%$. This number is lower than the percentage of women in the International Astronomical Union from all ESA Member States (24\%).

We compared our results with NASA statistics. Participation of women in NASA spacecraft science teams varies from none to just over 30\% [2]. The percentage has been increasing. However, this increase is more similar to a step function than a linear increase, with the pre-2000 average at $5.7 \%$ and post- 2000 at $15.8 \%$. This is well below the percentage of women in the field in the US, which has grown from $20 \%$ to $30 \%$ over the same time range. The ESA data are consistent with the NASA data, including the jump around the year 2000.

One of the main difficulties we encountered was to find the list of team members. An additional difficulty was to determine the percentage of female scientists in planetary science in Europe. We would like to encourage the planetary community as a whole, as well as international organizations, universities and societies to continuously gather statistics over many years. Detailed statistics are only the first step to closely monitor the development of achievement gaps and initiate measures to tackle potential causes of inequity, leading to gender inequalities in STEM careers. 
[1] Piccialli et al., submitted to ADGEO

[2] Rathbun, Julie A.: Participation of women in spacecraft science teams, Nature Astronomy, Volume 1, id. 0148 (2017). 